\title{
Was spricht dafür, das Deutsche als Naturwissenschaftssprache zu erhalten? [2001]
}

\section{Verehrter Herr Präsident,}

meine sehr geehrten Damen und Herren,

man hat mir gesagt: Präsident Kurt Mothes sprach noch deutsch, wenn er sich wissenschaftlich äußerte. Auf dem Symposion zu Ehren seines 100. Geburtstages, das vor kurzem hier in Halle abgehalten wurde, wurde dagegen bereits englisch gesprochen.

Die Sprachenwahl stand vielleicht nicht einmal zur Debatte. Was spricht dann dafür, das Deutsche als Naturwissenschaftssprache zu erhalten?

Die Frage ist bescheiden und nicht offensiv, sondern defensiv gemeint. Englisch ist zur Weltsprache in den Wissenschaften geworden, allen voran der Naturwissenschaften. Spricht gleichwohl etwas dafür, das Deutsche auf diesem Gebiet zu erhalten? Ist es sinnvoll, in den Naturwissenschaften neben einer universalen lingua franca über eine Vielfalt von Nationalsprachen $\mathrm{zu}$ verfügen? Ist absolute Einsprachigkeit ein Vorteil, oder ist Zweisprachigkeit, besser noch Mehrsprachigkeit auch in den Naturwissenschaften vernünftiger, vielleicht sogar notwendig? Dies war der Ausgangspunkt.

Das ursprünglich von mir vorgeschlagene Thema lautete entsprechend: „Einsprachigkeit, Zweisprachigkeit oder Mehrsprachigkeit. Was spricht dafür, das Deutsche als Naturwissenschaftssprache zu erhalten?“

Aus Halle kam die Frage, ob ich einverstanden sei, den langen Titel auf seinen zweiten Teil zu reduzieren. „Das ist doch der Kern Ihrer Aussage“, schrieb mir Präsident Parthier. Zugegeben: es ist der Kern. Aber inzwischen habe ich überlegt, ob das verkürzte Vortragsthema zweideutig sei. Denn als es auf dem erwähnten Symposion auftauchte und besprochen wurde, bemerkte ein Kollege aus NRW kurz und bündig: „Ich antworte auf diese Frage: Nichts spricht dafür, alles spricht für Englisch.“

Hatte der Westdeutsche die Frage so aufgefasst, als solle hier in Halle ein einseitiges Festhalten am Deutschen, eine Rückkehr zur Vergangenheit vorgeschlagen werden? Wird die Frage in Halle richtiger verstanden als in Köln, oder wird sie im Westteil der Bundesrepublik anders beurteilt als in ihrem Ostteil? Wollte der Kollege aus NRW tatsächlich sagen: für die Verwendung des Deutschen in den Naturwissenschaften gibt es überhaupt keinen Grund mehr, es ist auf diesem Feld überflüssig, „obsolet“, wie man zu sagen pflegt? Diese Auffassung ist keineswegs selten, und sie hat ihre Gründe. 


\section{Das Thema und mein Zugang}

Auf dem 25. Konstanzer Literaturgespräch des Buchhandels von 1985 wurde wohl zuerst eingehend über Deutsch als Wissenschaftssprache gesprochen. „Die Spitzenforschung spricht englisch - oder etwa nicht?“ lautete das Thema. Damals sagte Harald Weinrich einleitend:

Denn die Spitzenforschung spricht englisch, so hört man bei manchen akademischen Gelegenheiten sagen. Genauer ausgedrückt, man hört es gar nicht so oft sagen, es wird einfach getan, und verschiedene Wissenschaften im deutschen Sprachraum, die DDR teilweise ausgenommen, haben sich längst, ohne davon viel Aufhebens zu machen, auf englisch verabschiedet. Darüber wird in der wissenschaftlichen Öffentlichkeit, wiederum von wenigen Ausnahmen abgesehen, selten geredet, geschweige denn debattiert, so daß man sich fragt, ob es dafür überhaupt ein Problembewußtsein in der wissenschaftlichen Öffentlichkeit gibt [...] - ja, ist dieser Übertritt denn nicht einmal ein Schwellenbewußtsein wert? (Kalverkämper \& Weinrich 1986a: 18)

Im Januar dieses Jahres 2000 veranstaltete die Mainzer Akademie ein internationales Symposion zum Thema Deutsch als Wissenschaftssprache im 20. Jahrhundert, zu dem der Sprachwissenschaftler Friedhelm Debus, der Ingenieurswissenschaftler Franz Gustav Kollmann und ich eingeladen hatten. Ulrich Ammon, Sprachsoziologe, führte Statistiken vor (Ammon 2000). Der Befund überraschte allenfalls durch seine Eindeutigkeit: aus einer Weltsprache der Naturwissenschaften, die das Deutsche nicht nur in der Chemie von der Mitte des 19. bis in die 20er Jahre des 20. Jahrhunderts war, wird, zunächst aufgrund der Vertreibungspolitik der Nazis, dann während der 60er und 70er Jahre aufgrund der allgemeinen Entwicklung eine Randsprache, die keine Gewähr dafür bietet, international wahrgenommen zu werden. Zwischen 1980 und 1996 sinkt der Anteil der deutschen Sprache an naturwissenschaftlichen Publikationen von 3,5 auf 1,2 Prozent, der des Russischen von $11(10,8)$ auf $2(2,1)$ Prozent, steigt der des Englischen von $75(74,6)$ auf $91(90,7)$ Prozent.

Mit auffallender Entschiedenheit vertrat Wolfgang Gerok auf dieser Tagung die These „Die wissenschaftliche Medizin spricht englisch!“ Deutsch behalte in der ärztlichen Praxis und in der Ausbildung der Ärzte unverändert Bedeutung. „Aber wenn neue Erkenntnisse innerhalb der Scientific Community bekannt zu machen und kritisch zu diskutieren sind [...], ist Englisch und nur Englisch die Brücke der Kommunikation.“ (Gerok 2000: 231) Den oft zitierten Satz Harald Weinrichs, „Das Deutsche muß Wissenschaftssprache bleiben, nicht nur um der Zukunft der deutschen Sprache, sondern auch um der Zukunft der Wissenschaft willen“, halte er für unzutreffend, ja, er sei ihm nicht nachvollziehbar. Das Englische begünstige eine klare, präzise, knappe Darstellung, manche seiner Fach- 
ausdrücke wie ,patch-clamp-Technik‘, ,enhancer‘, ,splicing‘ seien kaum übersetzbar (Gerok 2000: 236).

Die „Sprachen der Wissenschaften an der Akademie der Wissenschaften der DDR“ wurden von Hartmut Schmidt (2000) behandelt. Aus seiner detaillierten Analyse ergab sich: gemessen an dem Aufwand, mit dem es durch die Akademie gefördert wurde, hatte das Russische nur einen bescheidenen Erfolg. „Die publizierenden Wissenschaftler der Akademie folgten von Anfang an dem internationalen Trend zur Publikation in englischer Sprache." Deutsch blieb die vorherrschende Publikationssprache, aber 1988 waren bereits 38 Prozent fremdsprachig, davon fast neun Zehntel englisch, kaum ein Zehntel russisch. In den Naturwissenschaften wurde der Zug zum Englischen in den siebziger Jahren deutlich erkennbar, Englisch lag aber 1978 in jeder Naturwissenschaft noch unter 50 Prozent. Zehn Jahre später, 1988, liegt es in der Physik über 60 Prozent, in der Mathematik, Biologie, Medizin über oder bei 50 Prozent, nur in der Chemie, die überhaupt eine beharrlich deutschsprachige Ausnahme bildet, bei kaum 30 Prozent (Schmidt 2000: 152 und 153: Übersichtstabelle I).

Es spricht also einiges dafür, dass nichts dafür spricht, das Deutsche auf dem Feld der Naturwissenschaften zu erhalten:

- Deren Gegenstand ist universal, weder der Mond noch die Physik sind ein deutsches Phänomen.

- Die Naturwissenschaftler sind eine internationale Such- und Verständigungsgemeinschaft.

- Distanzen der Sprache, des Raums und der Zeit erscheinen in der Kommunikation über Erkenntnisfortschritte in der Regel nur als Hindernisse.

- Raum und Zeit sind auf dem Erdball infolge der Kommunikationsentwicklung zusammengeschnurrt.

- Das Englische ist als Weltsprache durchgesetzt.

Bleibt allerdings die Frage: Ist die Sprache der Naturwissenschaften nichts als ein Vehikel der Verständigung unter Wissenschaftlern? In der Geschichte der neuzeitlichen Naturwissenschaft hat dieses ,nichts als‘ eine gewisse Wirkung gehabt, um 1800 beginnt es seine säkulare Wirkung zu entfalten. Der Mensch ist ,nichts als‘ eine Maschine, als ein chemisch-physikalisches Phänomen, als Materie. Die Beschränkung auf ,nichts als‘ ein Eines, die Einseitigkeit der Versuchsanordnung gehört zu den Erfolgsbedingungen neuzeitlicher Wissenschaftspraxis. Was bedeutet es, wenn man diese Erfolgsbedingung auch im Fall Naturwissenschaftssprache verabsolutiert?

Verehrte Kolleginnen und Kollegen, dieser Vortrag hier hat für mich etwas Unwahrscheinliches. Wenn man mir vor dreißig Jahren gesagt hätte, dass ich einmal vor Naturwissenschaftlern, noch dazu vor der Deutschen Akademie der 
Naturforscher Leopoldina in Halle über das Thema reden würde, was dafür spreche, das Deutsche als Naturwissenschaftssprache zu erhalten, hätte ich das für in jeder Hinsicht irreal gehalten. Ich bin Philologe, mein Arbeitsgebiet sind die Deutsche Literatur des Mittelalters und die Deutsche Sprache. Ich sah mich damals um nach einem Habilitationsthema. Auf dem Gebiet der Sprachwissenschaft herrschte die sogenannte ,Moderne Linguistik', die sich an Methoden und Schreibweisen der Naturwissenschaft anzulehnen suchte. Das war zu der Zeit obligatorisch, ich hatte größte Zweifel. Das szientistische Selbstverständnis der Sprachwissenschaft führte unser Fach, wie mir schien, auf dürre Weiden. Ich schlug mich in die Büsche und wählte ein Thema aus der Geschichte der Naturwissenschaft. Seit dem Ende der Studienzeit hatte ich hier zu lesen begonnen, zu exzerpieren. Hier las ich, was sich als Lieblingslektüre eignete, weiträumige, großartige Texte:

Linné, Systema naturae (1735)

Divisio et denominatio, Einteilung und Benennung werden das Fundament unserer Wissenschaft sein. Mineralia crescunt. Vegetabilia crescunt et vivunt. Anlmalia crescunt, vivunt et sentinunt. Die Steine wachsen. Die Pflanzen wachsen und leben. Die Tiere wachsen, leben und fühlen.

Linné, Philosophia Botanica (1751/1770)

$\S$ 213. Pflanzen, die der gleichen Gattung angehören, sind mit dem gleichen Gattungsnamen zu bezeichnen.

$\S 221$. Gattungsnamen, die aus zwei Wörtern bestehen, sind aus der botanischen Republik zu verbannen.

Goethe, Problem und Erwiderung (LA I, 9: 295)

Natürlich System, ein widersprechender Ausdruck. Die Natur hat kein System, sie hat, sie ist Leben und Folge aus einem unbekannten Zentrum, zu einer nicht erkennbaren Grenze. Naturbetrachtung ist daher endlos [...].

Goethe, Farbenlehre, didaktischer Teil, Schlußbetrachtung über Sprache und Terminologie (LA I, 4: 221)

$\S 751$ Man bedenkt niemals genug, daß eine Sprache eigentlich nur symbolisch, nur bildlich sei und die Gegenstände niemals unmittelbar, sondern nur im Widerscheine ausdrücke. Dieses ist besonders der Fall, wenn von Wesen die Rede ist, welche an die Erfahrung nur herantreten und die man mehr Tätigkeiten als Gegenstände nennen kann, dergleichen im Reiche der Naturlehre immerfort in Bewegung sind. Sie lassen sich nicht festhalten, und doch soll man von ihnen reden; man sucht daher alle Arten von Formeln auf, um ihnen wenigstens gleichnisweise beizukommen. 
Darwin, Entstehung der Arten (1867: 124)

Man kann figürlich sagen, die natürliche Zuchtwahl sei täglich und stündlich durch die ganze Welt beschäftigt, eine jede, auch die geringste Abänderung zu prüfen, sie zu verwerfen, wenn sie schlecht, und sie zu erhalten und zu vermehren, wenn sie gut ist [...].

Wir sehen nichts von diesen langsam fortschreitenden Veränderungen, bis die Hand der Zeit auf eine abgelaufene Weltperiode hindeutet, und dann ist unsere Einsicht in die längst verflossenen geologischen Zeiten so unvollkommen, dass wir nur noch das Eine wahrnehmen, dass die Lebensformen jetzt von dem verschieden sind, was sie früher gewesen sind.

Der Schlusssatz des Werks lautet (Darwin 1867: 565):

Es ist wahrlich eine grossartige Ansicht, dass der Schöpfer den Keim alles Lebens, das uns umgibt, nur wenigen oder nur einer einzigen Form eingehaucht hat, und dass, während unser Planet den strengsten Gesetzen der Schwerkraft folgend sich im Kreise geschwungen, aus so einfachem Anfange sich eine endlose Reihe der schönsten und wundervollsten Formen entwickelt hat und noch immer entwickelt.

Einstein, Prinzipien der Forschung, Rede zum 60. Geburtstag von Max Planck (1953: 143f.)

Keiner, der sich in den Gegenstand wirklich vertieft hat, wird leugnen, daß die Welt der Wahrnehmungen das theoretische System praktisch eindeutig bestimmt, trotzdem kein logischer Weg von den Wahrnehmungen zu den Grundsätzen der Theorie führt. Dies ist es, was Leibniz so glücklich als ,prästabilierte Harmonie‘ bezeichnete.

Nach unserer bisherigen Erfahrung sind wir nämlich zum Vertrauen berechtigt, daß die Natur die Realisierung des mathematisch denkbar Einfachsten ist.

Diese Lektüre war eine Erholung. Das war Sprache. Schriften der Naturwissenschaftler sind von Fall zu Fall Literatur, erstrangige Literatur. Ihr Werk steht voll von Sachen, ist das Fazit eines ergebnisreichen Blicks auf die Welt, knapp formuliert, anspruchsvoll und einfach auch angesichts komplexer Verhältnisse, lebendig, anschaulich und klar. Ihre Texte sind erregend für mich, wo diese Arbeiten Erkenntnisdinge berühren, das Verhältnis von Erkenntnis und Sprache, wo sie eine überlieferte Ausdrucksweise in Zweifel ziehen und über das sprachliche Handwerkszeug nachdenken.

Ich las sie damals nicht als Literatur. Die erkenntnispraktische Sprachreflexion des Naturwissenschaftlers ist für den Sprachwissenschaftler aufschlussreich, die Verbindung von Sprachgeschichte und Naturwissenschaftsgeschichte ist ergiebig, reizvoll im Einzelnen, aber auch ein wenig genutzter Schlüssel zur Geschichte der Neuzeit. Die Geschichte der Sprache ist ein Spiegel allgemeiner Entwicklungen, aber diejenige Wissenschaftsrichtung, deren den Erdball in der 
Neuzeit verändernde Wirkung außer Frage steht, hat in der Geschichte und Wissenschaft von der Sprache bisher keine annähernd angemessene Berücksichtigung gefunden. Ein Kreis weitreichender Fragen zeichnete sich ab: Was bedeutete der Übergang aus dem Lateinischen in die Landessprache für Wissenschaft und Gesellschaft? Wie steht es mit Gewinn und Verlust? Hing die Weltwirkung Darwins mit seinen zeitnahen bildhaften Schlüsselbegriffen „Struggle for Life“, „Natural Selection“ zusammen? Was verrät der Wandel der Bildfelder und Denkmodelle in den Naturwissenschaften, wenn man ihn vor dem Hintergrund der Epoche sieht? (vgl. Pörksen 1994a: 341; Pörksen 1986a: 126ff.) Eine solche weitreichende Frage ist auch, was uns hier beschäftigt. Sie hat nicht zuletzt eine geschichtliche Dimension. Fragen wir also, was für die Erhaltung des Deutschen in den Naturwissenschaften spricht, wenn man in die Geschichte schaut, in die Geschichte unserer allgemeinen Sprache und in die der älteren Naturkunde und neueren Naturwissenschaft.

\section{Blick in die Geschichte der deutschen Naturwissenschaftssprache}

Es gibt eine unausgesprochene Vorstellung von der normalen Einsprachigkeit des Menschen. Sie trifft nicht zu. Unser Sprachraum war zu keiner Zeit einsprachig, unsere Sprache wohl niemals eine homogene Einheit. Dass der Mensch von Natur ein „homo monolinguis“ ist, ein einsprachiges Wesen, ist ein Mythos des Nationalstaats, und dass es eine ursprünglich reine, unvermischte Sprache gab, ist in historischer Zeit nicht nachweisbar. (Die Inder sind so sprachbegabt, habe ich als Kind oft gehört, ihnen fliegen auf dem Markt mehrere Sprachen zu. Vielleicht sind sie gar nicht so speziell begabt, sondern haben nur nicht die europäische Theorie, die besagt, dass es eine Muttersprache und Fremdsprachen gäbe?)

Wenn man auf die Weltkulturen sieht, ist Zweisprachigkeit ein historischer Normalfall. Das gilt auch für die europäische Kultur. Das Gebiet, auf dem die menschliche Zunge sich deutschsprachig artikulierte, „theodisce“, wie es in einem latinisierenden Gelehrtenwort des 8. Jahrhunderts hieß - das Wort ,theodisc', aus dem dann ,diutisc', ,diutsch', ,deutsch' wird, bedeutete volkssprachig, landessprachlich, laiensprachlich -, das Gebiet also, wo die menschliche Zunge sich auf unsere laiensprachliche Weise „deutsch“ artikulierte, war vom 8. bis zum 18. Jahrhundert ein Gegenüber von Deutsch und Latein, genauer gesagt von überwiegend mündlich gebrauchten deutschen Dialekten und einer lateinischen Schriftkultur (vgl. Pörksen 1999b). Die Sprache der Kirche und Klöster, der späteren Universität, der Poesie, des Rechts und der Diplomatie waren lange und dann 
abnehmend lateinisch. Ein solches Gegenüber von Laiensprache und Mandarinensprache kennen wir aus Arabien und Indien, Indonesien, Tibet oder China.

Naturkundliche und heilkundliche Handschriften beginnen im Mittelalter. Wenn man sich die alten naturkundlichen oder heilkundlichen Texte anschaut, frühe Zeugnisse der deutschen Sprache, die im 12. Jahrhundert beginnen, so geht es einem zunächst oft so: Sie haben etwas holprig Unmittelbares, man glaubt, einen mittelhochdeutschen Originalton zu hören, einen volkssprachlichen, der auf mündlicher Überlieferung beruht, und dann ist es doch wieder die Übersetzung oder Kompilation aus dem Lateinischen. Der lateinische Prägestock hat sich tief in unsere Grammatik, den Satzbau und Stil, in unseren Wortschatz eingegraben. Das gilt nur unter anderem für die Wissenschaften. Unsere deutsche Schriftsprache insgesamt ist nicht so sehr aus der mündlichen deutschen Sprachkultur hervorgewachsen, wie man spontan annehmen möchte, sie entstand eher im Bruch mit ihr, angelehnt, als „Lehnbildung“ an das Lateinische. Noch, was der erste deutsche Träger des Literaturnobelpreises schrieb, der Althistoriker Theodor Mommsen, ist eigentlich Latein, liest sich wie die deutsche Rückseite einer lateinischen Tapete.

Latein und das hinter ihm stehende griechische Hintergrundsmassiv haben also nicht nur die Geschichte unserer älteren Wissenschaftssprachen, speziell die der Naturwissenschaftssprachen geprägt, sondern unsere Sprache insgesamt. Ein Unterschied zum Englischen besteht darin, dass bei uns viel mehr Ausdrücke aus den klassischen Sprachen ins Deutsche übersetzt wurden, auch in den Wissenschaften. Konrad von Megenberg (1358) nannte den Horizont den ,ougenender‘, die Mondfinsternis ,scheinprechen' (Scheingebrechen), für Dürer war die Diagonale eine ,Zwerchlinie“ (Querlinie), die Ellipse eine ,Eierlinie‘ und die Hyperbel eine ,Gabellinie‘, Kepler sagte in seiner Messekunst Archimedes für Segment auf gut schwäbisch ,Schnitz‘ und für Parallele ,Gleichlaufende‘. Diese Wörter gingen unter. Manche Neubildungen von Dürer und Kepler nahm Christian Wolff später in sein Mathematisches Lexikon (1734) auf (vgl. Pörksen 1998: 198). Das Englische blieb viel stärker lateinisch durchsetzt. Bei Chaucer hat man gezählt, dass 38 Prozent seiner Wörter dem Lateinischen entstammen, und man kann heute z. B. beim Lesen einer englischen Sprachgeschichte feststellen, dass auf einer Seite die Hälfte des Wortschatzes im Ursprung lateinisch ist. Im Englischen sind der romanische und germanische Sprachstamm zusammengewachsen; es eignet sich auch deshalb, nicht nur der einfachen Grammatik wegen, als Universalsprache.

Unser Sprachraum war also 1000 Jahre zweisprachig, lateinisch und deutsch, im 17. und 18. Jahrhundert kam in erhöhtem Maß das Französische hinzu, man kann von Dreisprachigkeit und von einer recht weitgehenden Sprachmischung reden. Sie wurde parodiert, Sigmund von Birken dichtete 1645: 
Ich bin nun deschargirt von dem maladen Leben.

Mir hat der Maur facon genug disgousto geben.

Wo Einfalt avancirt, und Unschuld mit raison,

Die retrogarde hat, da ist die Sache bon.

Wenn gestern auf einem Wahlplakat „Keep Kohl“ stand und man heute in der Bonner U-Bahn liest, „Clean ist wieder in“, wenn ein Jugendlicher gute „connections“ zu seiner „family“ hat und ein Wissenschaftler einen „approach“ macht und ein „handout“ verteilt, hat das eine lange lateinisch-deutsche, französisch-deutsche Vorgeschichte.

Der Adel sprach und schrieb französisch, aber der französische Einfluss erfasste sehr bald alle Bereiche, nicht nur Küche und Liebe, sondern auch die Verhandlungssprache in gelehrten Körperschaften, in Akademien z. B. „Bei Entlehnungen kommt es vor allem auf das Prestige an“, schrieb der Sprachwissenschaftler Stephan Ullmann. Was dem breiten Entlehnungsvorgang zugrunde lag, war das Bewusstsein eines Kulturgefälles zwischen Deutschland und Frankreich, das Gefühl einer Inferiorität: das Prestige der französischen aristokratischen Bildung und Kultur.

Das Gegenüber des Deutschen und Lateinischen und die sogenannte deutsch-französische ,Sprachmengerei', von der ein Autor wie Christian Thomasius noch Ende des 17. Jahrhunderts meinte, sie sei irreversibel, löste zu jener Zeit eine Gegenbewegung aus, die ungemein erfolgreich war. Hier liegt der Ursprung der deutschen Wissenschaftssprache.

Der vermutlich wirksamste und sicher vorausschauendste Aufsatz, die deutsche Sprache betreffend, stammt aus dieser Zeit, er wurde um 1700 dreimal umgeschrieben und hat den Titel Unvorgreifliche Gedanken betreffend die Ausübung und Verbesserung der deutschen Sprache. Er beginnt:

Es ist bekannt, daß die Sprache ein Spiegel des Verstandes ist und daß die Völker, wenn sie den Verstand hoch schwingen, auch zugleich die Sprache wohl ausüben, welches der Griechen, Römer und Araber Beispiele zeigen. (Leibniz 1983: 5)

Der Verfasser, Gottfried Wilhelm Leibniz, diagnostiziert von einem historischen Standpunkt aus - das Vorbild der Académie française und der französischen Sprache vor Augen - fast so etwas wie eine Krise der deutschen Sprache. Auf der einen Seite meint er die ,Sprachmengerei'; sie erschien ihm als eine Form der Unterwerfung. Das Deutsche sei während des 30-jährigen Krieges „in die Rappuse“ gegangen. (1700, bei der Gründung der Berliner ,Societät", der späteren ,Berliner Akademie der Wissenschaften', schrieb er in deren Generalinstruktion hinein, die Akademie werde Sorge tragen, dass der Selbststand unserer wahren deutschen Hauptsprache gewahrt werde, damit nicht ungereimter Mischmasch 
und Undeutlichkeit daraus entstehe. - Deren erste Verhandlungssprache ist dann aber Französisch gewesen.)

Auf der anderen Seite meint Leibniz die Sprachentrennung zwischen Gelehrten und Laien, das Latein als Barriere allgemeiner Bildung. Sie beschäftigt ihn seit langem.

Die Wissenschaft ist dem Licht gleich, und es ist ein allgemeines Interesse, daß es auf alle einzelnen ausgegossen sei. (Leibniz 1916a: 86)

Er entwickelt in diesem sprachplanerischen Meisterwerk ein Wörterbuchprogramm, das bis heute nicht eingeholt ist, z. B. die Idee eines internationalen Fachsprachenlexikons. Das Deutsche sei eine buchstäblich unkultivierte Sprache, in manchen praktischen, konkreten Fächern sei es zwar flächendeckend ausgebaut, aber dieser Ausbau fehle auf allen Gebieten der höheren Abstraktion und berechtigter Ansprüche an ein Kulturvolk: auf den Gebieten der Regierungskunst, Ethik, der Wissenschaften, Logik, Metaphysik werde diese Sprache - er meint es buchstäblich - nicht ausgeübt. Ausübung meint Ausbau. Anders als die Sprachgesellschaften, die auf Poesie und Übersetzungen von Poesie setzten, setzt er auf jenes Schrifttum, das einen Kern in sich hat. Er schlägt die Ausarbeitung eines Kanons deutscher Sachprosa vor. In seinen Augen ist es vor allem die Non-fictionLiteratur, die eine Sprache voranbringt. Leibniz schrieb in der Regel Latein und Französisch. Seine Unvorgreiflichen Gedanken wurden erst 1717, ein Jahr nach seinem Tod veröffentlicht.

Das Interessante für unsere Leitfrage ist: das, was hier gefordert wurde, geschieht. Es setzt um 1700 entschieden ein, am Ende des 18. Jahrhunderts gibt es eine flächendeckend und differenziert ausgebaute öffentlich durchsichtige Gemeinsprache. Sprachkritik ist zum Faktor der Sprachgeschichte geworden. Es existiert kein Staat, keine Akademie, keine zentrale Instanz, die verantwortlich zu machen wäre: Kultursprache Deutsch. „[...] es war kein Wunder“, schreibt der englische Sprachhistoriker Eric A. Blackall (1966: 2) einleitend. „Es war vielmehr ein stetiger und oft durchaus bewußt vorangetriebener Entwicklungsprozeß, an dem sehr unterschiedliche Kräfte teilhatten.“

Es ist nicht ganz ohne historische Pointe, wenn die Frage, ob etwas dafürspricht, das Deutsche in der Naturwissenschaft zu erhalten, hier in Halle gestellt wird. Denn an den Namen Halle knüpft sich der Durchbruch der deutschen Wissenschaftssprache an unseren Universitäten des 18. Jahrhunderts. Es war der Vorreiter. Christian Thomasius wurde, nachdem er als junger Mann in Leipzig mit diesem Versuch gescheitert war, an die Ritterakademie in Halle berufen und hat an der zur Universität gewordenen Akademie die deutschsprachige Vorlesung durchgesetzt. Christian Wolff hat hier den Denkstil neuzeitlicher Naturwissenschaft ins Deutsche übertragen, d.h. er hat aus dieser Sprache ein brauchbares 
Instrument methodischen naturwissenschaftlichen Denkens und Darstellens gemacht. Ich brauche das in Halle, wo Ulrich Ricken (1989) gearbeitet hat, nicht auszuführen. Bei dem Sprachenwechsel vom Lateinischen zum Deutschen ging es allerdings um viel mehr als den Austausch eines Verständigungsmittels. Thomasius definierte einen neuen Bildungsauftrag der Universität. Er wollte das „aristotelische Orgelwerk“, den traditionsgebundenen Lehrkanon ersetzen durch ein an das Licht der Vernunft gebundenes, freies, ins praktische Leben wirkendes Forschen und Lehren. Sein Bildungsideal war der vollkommene und weise Mann, „den man in der Welt zu klugen und wichtigen Dingen brauchen kann“. Der Sprachenwechsel der Universität war ein Funktionswandel, ein Austausch der Denkstile. Jürgen Schiewe hat in seiner Schrift über Die Universität Freiburg zwischen Latein und Deutsch (1996), in der er Basel und mehr noch Halle zum Vergleich heranzieht, genau dies betont: Sprachenwechsel als Funktionswandel der Wissenschaft und Austausch der Denkstile.

Eine deutsche Naturwissenschaftssprache, die neue wissenschaftliche Inhalte zuerst auf deutsch publizierte, hat es weniger als 200 Jahre gegeben, vom Ende des 18. bis in die sechziger Jahre des 20. Jahrhunderts. Diese Periode hat eine zu recht bewunderte Sachprosa hervorgebracht, von Blumenbach, Kielmeyer, Goethe, Humboldt über Helmholtz, du Bois-Reymond, Haeckel bis zu Einstein, Heisenberg, von Frisch, Portmann, Lorenz usf. Diese Sprache war ein Vehikel der Wissenschaft. Zugleich war sie Literatur, ein Kulturphänomen. Der Naturwissenschaftler trat ein in die Rolle des allgemeinen Weltinterpreten, machte sie dem Theologen und Philosophen streitig.

Oder er orientierte einfach. Wissenschaft wurde allgemein durchsichtig und zugänglich, sie bekam einen demokratischen Zug.

Die öffentlichen Vorträge der Wissenschaftler, mit denen Justus Liebig oder Helmholtz hervortraten, hatten noch eine weitere Funktion: Populärwissenschaft diente dazu, die Gesellschaft für die neue Naturwissenschaft zu gewinnen, ihr einen Raum, Anerkennung, Förderung zu verschaffen, sie institutionell zu verankern. - Brechen wir den historischen Ausflug hier ab und kehren zur Ausgangsfrage zurück:

Was spricht dafür, nach dem jüngsten Sprachenwechsel zum Englischen das Deutsche in den Naturwissenschaften zu erhalten?

Spricht nichts dafür, alles für Englisch? Geht es bei dem Übergang der deutschen Naturwissenschaft zum Englischen um mehr als den Wechsel zu einer bequemen Universalsprache? Steht wiederum ein Wissenschaftskonzept auf dem Spiel? Ein neues Paradigma? Hat dies Paradigma nationale, hegemoniale Züge oder ist es eine Universale? Steht die Sprache selbst auf dem Spiel?

In Marburg gab es noch vor kurzem ein Physikalisches Institut, das seine wissenschaftliche Literatur bis 1960 in der Veranda deponiert hatte. Dieser Biblio- 
theksteil hieß ,Steinzeit‘, die Veranda war die Steinzeit. Wir haben uns eben mit der Älteren Steinzeit beschäftigt.

Mir scheint, die Naturwissenschaftler stehen vor der Frage, in welchem Sinn sie das Deutsche aufheben wollen. Hegel war bekanntlich fasziniert von den verschiedenen Bedeutungen, die das Wort ,aufheben“ in unserer Sprache annehmen kann:

- Wollen sie das Deutsche aufheben im Sinn von aufhören lassen, ihm ein Ende machen?

- Wollen sie es aufheben im Sinn von aufbewahren, erhalten?

- Oder ist etwas Drittes denkbar, das beides verbindet: könnte man es aufheben, indem man es auf eine neue Stufe hebt, es verändert aufhebt?

Erlauben Sie im letzten Teil des Vortrags eine Reihe Fragen, Hypothesen und Thesen.

\section{Fragen, Hypothesen und Thesen zur Einsprachigkeit, Zweisprachigkeit, Mehrsprachigkeit der Naturwissenschaften}

1.

Unsere öffentliche Umgangssprache, die deutsche Bildungssprache wird im anbrechenden Jahrhundert vermutlich eine Prägung durch das Englische erfahren, die der 1000-jährigen Prägung durch das Lateinische und der 200-jährigen durch das Französische vergleichbar ist. Der Wortschatz wird sich erweitern - sei es durch Übernahme und Assimilation entlehnter Vokabeln, sei es durch ihre deutsche Lehnübersetzung. Unsere Sprache wird sich europäisieren, internationalisieren.

Die dreibändige Geschichte der deutschen Sprache vom 15. bis zum 20. Jahrhundert, die Peter von Polenz (1991/1994/1999) im vergangenen Jahrzehnt vorgelegt hat, ein vorzügliches Werk, hat gezeigt, welche Herausforderung das Lateinische und Französische in unserer Geschichte gewesen ist - und welchen Gewinn, welche Horizonterweiterung und Kultivierung die Auseinandersetzung erbracht hat. Die Assimilationskraft der Sprache ist groß. „Ich verfluche allen negativen Purismus“, hat der alte Goethe gemeint. „Die Gewalt einer Sprache ist nicht, daß sie das Fremde abweist, sondern daß sie es verschlingt. Ich verfluche allen negativen Purismus, daß man ein Wort nicht brauchen soll, in welchem eine andere Sprache Vieles oder Zarteres gefaßt hat.“ (Hecker 1907: 979f.) Es käme also 
auf den guten Magen an. In der überkommenen ausgezeichneten deutschen Naturwissenschaftssprache haben wir einen erstklassigen Magenbitter.

\section{2.}

Die Sonderstellung von Wissenschaftssprachen in der Gesellschaft, Zweisprachigkeit in diesem Sinn, ist ein historischer Normalfall. Dass die heutigen Naturwissenschaften über eine Weltsprache wie das Englische verfügen, erscheint mir als großer Vorzug, der zu Recht begrüßt wird, aber auch als ein erheblicher Einschnitt, aus dem Konsequenzen zu ziehen sind. Wir begegnen der Zweisprachigkeit zumeist in zwei Formen: einmal als Pendeln der Gelehrten zwischen zwei Sprachen, die Sprachwissenschaftler sprechen von ,Bilingualismus‘, der in vielen Fällen zur Sprachmischung, zur regulären Mischprosa, aber auch zu Kauderwelsch und Missingsch, zum unentbehrlichen ,Laborslang' führt, und dann begegnen wir ihr als Verteilung der beiden Sprachen auf verschiedene Bereiche, dem Deutschen in der Praxis, dem Lateinischen oder Französischen oder Englischen in der Wissenschaft. Die Sprachwissenschaftler sprechen von ,Diglossie‘. Die deutsche Naturwissenschaftssprache ist seit Jahrhunderten speziell charakterisiert durch eine geregelte Sprachmischung, man könnte auch sagen, eine ,sprachinterne Diglossie‘. Das Fachvokabular ist lateinisch, griechisch und neuerdings englisch, der Rest deutsch. Ich nenne diese sehr praktische Einrichtung den ,Fachwerkstil‘ (Pörksen 1994b: 37ff.).

\section{3.}

Naturwissenschaftler haben eine Neigung, Sprache für unwichtig zu halten. Sie erscheint ihnen oft als bloßes Instrument, durchsichtig auf den Gegenstand hin wie Wasser. Sie halten es gern mit dem Kollegen Faust: „Ich kann das Wort so hoch unmöglich schätzen.“ Dazu passt, dass sie im Vortrag frei sprechen. Die Sache stellt sich für sie durch das eine oder andere Wort von selber dar. Geisteswissenschaftler oder Kulturwissenschaftler halten sich eher an das Manuskript. Für sie sieht es so aus, als habe die Sprache ein Eigengewicht, als sei sie am Aufbau ihrer Gegenstände beteiligt. Das Medium bricht den Gegenstand, die beiden sind kaum zu trennen. Ich bin auf dieser Seite, ich bin Philologe. 


\section{4.}

In den Naturwissenschaften hat sich seit langem eine ,Sprache‘ ausgebreitet, die noch eindeutiger international ist als das Englische und nur metaphorisch als Sprache bezeichnet werden kann. Dazu gehören: Abkürzungssymbole, Kurzwörter, Formeln; Zeichnungen, Pfeile, Schemata und Figuren; Modelle und Schaubilder; die Visualisierung von Daten durch Tabelle und Diagramm, Photographien und Schirmbild füllt die Seiten. Diese Arbeits- und Darstellungsmittel haben so zugenommen, dass man vom sprachlosen Gelehrten spricht. Der Eindruck des Standardisierten, des Rasters, ist dabei in vielen Darstellungen stark. Auch die sprachlose Sprache lenkt, wie mir scheint, den Blick, ist ein Sehrohr, ein Perspektiv. Daneben gibt es die ,Sprache‘ der Mathematik. Beides soll hier nur gestreift werden. Es unterstreicht den internationalen Charakter der Naturwissenschaften.

\section{5.}

Was als englische Weltsprache in Gebrauch ist, nennt Wolf Lepenies Englisch II. Das ist nicht nur witzig, es ist außerdem eine nützliche Unterscheidung. Englisch I hat einen sehr differenzierten Wortschatz, was jeder merkt, der englische Gedichte liest, es verfügt über eine komplexe Grammatik und ist in der Lage, den Begriffsapparat und die Nomenklatur eines Faches kritisch zu durchleuchten und vielfältig zu variieren. Es hat einen ganzen Kulturraum im Rücken. Englisch II ist eine Funktionssprache, die auf einen verkleinerten Wortschatz, eine bescheidene Grammatik und das im jeweiligen Zusammenhang erforderliche Fachvokabular zurückgeschnitten ist. Sie erfüllt ihren Zweck, ist aber wenig inspirierend.

\section{6.}

Der Gebrauch dieser schmalen Funktionssprache ist nicht nur mit den sprachlosen, internationalen Darstellungsmitteln von Mathematik und Visualisierung verbunden, sie ist zugleich die Verkehrssprache der Kommunikationsgeräte, die seit der jüngsten elektronischen Medienrevolution die Arbeitsweise der Naturwissenschaften verändert hat. Englisch ist die Schlüsselsprache der Computertechnik, die Anschlusssprache des naturwissenschaftlichen Gesprächs, des Datenflusses, der Kooperation rund um den Erdball. Wir haben vorhin überlegt, ob mit dem Übergang zum Englischen ein Funktionswandel der Universität, ein Umbruch des Denkstils einhergehen könnte. Jürgen Schiewe (2000: 103f.) formulierte auf dem erwähnten Mainzer Symposion dazu folgende Überlegungen: 
1. Der Übergang vom Lateinischen zum Deutschen stand mit dem Austausch des scholastischen Denkstils durch ein aufklärerisches Nützlichkeitsdenken und dem Wandel der Universität von einer autonomen Korporation zu einer Staatsuniversität in Verbindung.

2. Der Übergang vom Deutschen zum Englischen könnte ebenfalls mit einem Austausch der Denkstile und einem Funktionswandel verbunden sein. Als Austausch der Denkstile kommt ein Vorgang in Betracht, der mit folgenden Stichworten anzudeuten wäre: Ein eher historischer, diskursiver, erklärender Wissenschaftsbegriff wird durch einen system- und theoriegebundenen Wissenschaftsbegriff einerseits und einen auf wissenschaftliche und technische Anwendbarkeit basierenden Wissenschaftsbegriff andererseits ersetzt.

3. Die Funktion der Universität, als maßgeblicher Ort für Wissenschaft, nützlich für Staat und Gesellschaft zu sein, wird abgelöst durch die Funktion, im Dienste der Wirtschaft produktiv zu sein und sich in einem weltweiten virtuellen Kommunikationsraum zusammenzuschließen.

4. Die neuen Stichworte für die englischsprachige Universität lauten: Wirtschaft und Virtualität. Beides ist international, beides ist überwiegend englischsprachig. Kein Wunder also, wenn auch die Universität, die sich zunehmend in eine Gemeinschaft mit der Wirtschaft begibt und die den Sprung in die Virtualität schaffen möchte, sich in ihrem wesentlichen Betätigungsfeld, der Wissenschaft, englischsprachig gibt. Wiederum wäre der Sprachenwechsel nur eine Folge, die sich konsequenterweise aus anderen Erscheinungen, aus der Globalisierung von Wirtschaft und Medien, ergibt.

\section{7.}

Englisch II ist brauchbar auf dem Feld der Wissenschaftsfabrikation, aber wie förderlich ist es bei produktiver Forschungsarbeit? Es reicht aus für die Beschreibung der Milbenarten in einigen Gewässern am Südhang des Himalaya. Wie aber, wenn das Klassifikationssystem, die Kriterien der Artenunterscheidung fraglich werden? Wenn eine neue Theorie des Gegenstands gefragt ist, unbekanntes Gelände betreten wird? Dann denken wir in der inneren Umgangssprache, nehmen ihre Assoziationen, die in uns lebende Bilderwelt, Redensarten, zu Hilfe, strecken die Fühler aus in alle möglichen Ecken unserer Sprache, unseres und fremder Kulturräume. Die Beschränkung auf Englisch II wäre da vermutlich ein ziemlicher Nachteil, eine Scheuklappe. Wer englisch spricht und denkt, hat einen Vorteil, weil er über beide Idiome verfügt, sich in beiden Registern bewegt. Wer deutsch spricht und denkt und nur über Englisch II verfügt, dem fehlt im Englischen der Assoziationsraum, über den der native speaker verfügt. Wenn die Inhalte von Englisch II nicht auf deutsch versprachlicht vorliegen, nicht übersetzt sind, stellt sich aber auch der entsprechende deutsche Assoziationsraum unvollständig ein. Er fehlt also hier wie dort. Das würde, falls es zutrifft, bedeuten:

- Die Situation verlangt, dass der deutsche Sprecher ein vorzügliches Englisch I erwerben muss. 
- Sie legt darüber hinaus nahe, dass Englisch II ins Deutsche übersetzt vorliegt, damit sich bei ihm der reichere Assoziationsraum der eigenen Sprache einstellt. Ein wissenschaftliches Werk, das diesen Namen verdient, sei es auf englisch oder auf deutsch, kann vermutlich erst dann entstehen.

8.

Wenn jemand die Zusammensetzung der Atmosphäre in 10.000m Höhe zu erkunden und zu beschreiben hat, genügt vielleicht Englisch II. Wenn aber eine Gruppe von Wissenschaftlern aus fünf verschiedenen Ländern und fünf unterschiedlichen Disziplinen zusammenarbeitet, um sich eine Vorstellung von der Klimaentwicklung zu bilden, lässt sich folgendes beobachten (Joachim Nettelbeck, der Sekretär des Berliner Wissenschaftskollegs, hat es mir berichtet): bei dem Versuch, ihre Beobachtungen, Daten, Vermutungen zu einem Gesamtbild, einem dynamischen Modell zusammenzufügen, greifen sie zu zunächst schlichten Bildern, groben Metaphern, versuchen, ob sie passen, ändern sie ab. Sie greifen zurück auf den ihnen naheliegenden Sprachraum. Dabei zeigt sich, dass man in verschiedenen Sprachen unterschiedliche Bildfelder bevorzugt (vgl. Nettelbeck 2000: 105ff.). Das ist ein Vorteil.

Mehrsprachigkeit scheint überhaupt ein Vorteil für die Forschung zu sein. Die Zahl der Gesichtspunkte wächst. Die Funken springen vielleicht um so besser, je weiter die Disziplinen und Sprachen auseinanderliegen. Es ist etwas dran an Wilhelm von Humboldts Idee, dass Sprachen ,Weltansichten' sind (Humboldt 1963, III: 19f.).

\section{9.}

Wissenschaftssprachen sind auch Ausdruck von Wissenschaftskulturen. Ein englisches Buch kann man nicht nur lesen, sondern auch hören, es ist konkreter, knapper und gemeinsprachlicher, als wir es gewohnt sind, gelegentlich witzig. Ihm fehlt ein gewisser bürokratischer Ernst.

Russels fabelhafte History of Western Philosophy würde bei uns nicht geschrieben werden.

Verschiedene Wissenschaftskulturen fordern einander heraus, regen einander an. Der Kunsthistoriker Erwin Panofsky hat bekannt, dass ihm erst die Emigration in die USA die Zunge gelockert und ihn ermutigt hat, Bücher über ganze Meister oder Epochen zu schreiben, statt ein Dutzend Spezialaufsätze (vgl. Pörksen 1994a: 304f.). 
Wissenschaftskulturen, Wissenschaftssprachen regen einander an, Verschiedenheit ist so nützlich wie Verständigung, sie bereichert.

Kurz: Wer als Naturwissenschaftler nur über Englisch II verfügt, ist arm dran. Er braucht, wenn er im Wettstreit mithalten und sich behaupten will, ein in Leibniz' Sinn flächendeckendes, breites Englisch und eine naturwissenschaftlich ausgeübte Muttersprache.

Schon um der Forschung willen scheint mir die Übersetzung und ständige Ausarbeitung einer deutschen Wissenschaftssprache nötig zu sein.

Die Argumente werden noch leichter erkennbar und schlagender, wenn man die öffentliche und kulturelle, nationale, die politische und gesellschaftliche Bedeutung der Naturwissenschaften im Auge hat.

\section{0.}

Englisch ist nicht einfach, wie es das tote Latein war, eine Universalsprache, sondern auch eine nationale Hegemonialsprache. Es ist mit einer bestimmten Wissenschaftskultur verbunden, einer Tradition, auch mit gewissen wissenschaftlichen Modellen und Paradigmen. Seiner ausschließlichen Herrschaft, die dazu führen kann, dass Wichtiges übersehen wird und nicht zur Geltung kommt, ist nur durch Gegenaktivität zu begegnen, durch aktive Zufuhr aus anderen Denkkreisen.

\section{1.}

Innerhalb unserer Gesellschaft fallen die einschneidenden Zukunftsentscheidungen der Regierung und des gesetzgebenden Parlaments auf Gebieten, deren jüngste Entwicklungen nur noch auf Englisch zugänglich sind. Naturwissenschaften und Medizin, Ökonomie und Technik sind unsere Großmächte. Die Naturwissenschaften sind ein Kulturphänomen, zugleich aber auch der stärkste Motor gesellschaftlicher Veränderungen. Die Beratung dessen, was hier vorgeht, bedarf einer öffentlich durchsichtigen Sprache.

\section{2.}

Unsere allgemeine öffentliche Sprache bleibt zurück, wenn die modernen Naturwissenschaften nicht in ihr ausgearbeitet werden, wenn sie in dieser Richtung nicht ausgebaut wird. Dann tritt das ein, was Leibniz bemängelte. Das Deutsche, Dänische, Schwedische usw. gewinnt den Status eines Dialekts. 
Sicher wäre es unnötig, alles, was in Englisch II vorliegt, zu übersetzen, aber wenn das, was neu und wichtig ist und einen neuen Blick auf die Welt wirft, nicht eingedeutscht wird, verengt sich der Horizont unserer Sprache. Dass hier durch Zeitschriften, den Wissenschaftsteil der Zeitungen, die Übersetzung englischer naturwissenschaftlicher Werke Enormes geleistet wird, soll in keiner Weise bezweifelt werden. Es wäre aber von großem Wert, wenn die Naturwissenschaftler selbst sich beteiligen und die Auswahl treffen würden, wenn sie das, was nach einem Werk aussieht, auch in deutscher Sprache vorlegen würden. Dann hätten wir ein Weiterleben der Wissenschaft als Literatur. Die Naturwissenschaft als Literatur aufzuheben im Sinn des Aufhörenlassens, wäre ein unschätzbarer Verlust, sie - in gezielter Auswahl - aufzuheben im Sinn des Erhaltens hieße, die öffentliche Begriffssprache auf den Stand der Dinge zu bringen. Die produktiven Forscher sind dazu am geeignetsten.

\section{3.}

Populärwissenschaftlich im letzten Sinn, öffentliche Durchsichtigkeit der Naturwissenschaften, von den Forschern selbst besorgt, verankert ihre Arbeit in der Öffentlichkeit, macht die Öffentlichkeit $\mathrm{zu}$ deren Anwalt. Aus diesem Grund haben viele Naturwissenschaftler seit dem 19. Jahrhundert sich an zugänglichen, allgemeinsprachlichen Darstellungen versucht.

\section{4.}

Auf den Einwand, es sei nicht mehr möglich, was die heutige Naturwissenschaft hervorbringt, gemeinsprachlich darzustellen, antworte ich - da ein Exkurs darüber zu einem zweiten Vortrag würde - mit dem Wort eines Biologen. Bernhard Hassenstein (1979: 238) formuliert und begründet in seinem herrlichen Münchener Vortrag Wieviele Körner ergeben einen Haufen? Bemerkungen zu einem uralten und zugleich aktuellen Verständigungsproblem einen sensationellen Lehrsatz:

Die Umgangssprache ist nicht notwendigerweise vage, schillernd oder ungenau; das ist lediglich die ohne Könnerschaft gehandhabte Umgangssprache. Der Möglichkeit nach ist die Umgangssprache in der Darstellung der Wirklichkeit von beliebiger Präzision.

Der Satz ist in den Augen eines Sprachwissenschaftlers beweisbar. Die Leistungsfähigkeit der Umgangssprache wird speziell von deutschen Wissenschaftlern maßlos unterschätzt. Sie reicht weit. 


\section{5.}

Ihr Gebrauch bedeutet, dass die naturwissenschaftliche Weltdeutung von selbst in Berührung gerät mit der Alltagsdeutung der Welt. Es entsteht eine neue Perspektive, aus der das interne Wissenschaftsereignis gesehen wird: es wird in seiner öffentlichen Bedeutung erkennbar, in seiner Konsequenz für das öffentliche Weltbild wie für eine mögliche Veränderung der Welt. Wenn die Naturwissenschaftler hier nicht das Wort nehmen, kann es dahin kommen, dass von Staatsmännern und Feuilletonisten lächerliche Weltenten verbreitet werden wie z.B. die, das menschliche Genom sei entschlüsselt. Wir sind von Entwicklungen der Naturwissenschaften in unseren Lebensmöglichkeiten und Sterbensmöglichkeiten betroffen wie von keiner anderen Wissenschaft. Soll die Öffentlichkeit hier mitreden, - sie soll und tut es - dann braucht sie klare Vorstellungen und die Wahrheitsliebe, die Zivilcourage der Wissenschaft.

\section{6.}

Die Regierung und das gesetzgebende Parlament braucht ein klares, offenes, differenziertes Bild. Die Politikberatung erfolgt öffentlich, in den Medien, und intern. Beide Formen sind auf die öffentliche Durchsichtigkeit der naturwissenschaftlichen Entwicklung angewiesen: auf ihre deutsche Versprachlichung. Was ansteht, sind Fragen vom Umfang Energiewirtschaft, Gentechnik, Wasserwirtschaft.

\section{7.}

Von einer deutschsprachigen Naturwissenschaft als Literatur profitieren die Dichter. Auf dem Weg über die Dichtung werden neue Begriffe, Ausdrücke, Vorstellungen der Naturwissenschaft vielleicht am behutsamsten assimiliert und der allgemeinen Sprache einverleibt. Die vorangegangene Autorengeneration, Musil, Jahnn, Broch, Thomas Mann, Werfel, beide Jüngers haben durchaus noch neuere Naturerkenntnis und Beschreibung in ihr Werk integriert. (Thomas Mann hat eine ganze Passage bei Portmann stiebitzt.) - Das ist sehr viel weniger geworden; um so interessanter, dass sich eine jüngere Generation, Autoren wie Durs Grünbein und Raoul Schrott, gerade dieser Sache annimmt. 


\section{8.}

Ich verzichte auf Bemerkungen zur Praxis, zu der Frage, wie die ,Ausübung“ englischer und deutscher Naturwissenschaften an den Universitäten zu erreichen sei, wie Englisch und Deutsch auf die verschiedenen Textgattungen, Einführung, Vorlesung, Handbuch, Dissertation, Zeitschrift etc. zu verteilen wäre. Erlauben Sie aber einen Vorschlag zur Güte: Die Naturwissenschaftler brauchen Zeit, wenn sie ihre Hauptsachen ins Deutsche bringen wollen. Die sollte ihnen institutionell und großzügig eingeräumt werden. Sehr schön wäre ein Englisch-Deutsch-Kolleg der Naturwissenschaften, ein Kolleg, wo kreative Naturwissenschaftler die Chance erhielten, ein Jahr lang ein Werk auf deutsch auszuarbeiten. Dann würden beide profitieren: die Wissenschaft und die Öffentlichkeit. Ich bitte zu bedenken:

Die Umgangssprache ist von beliebiger Präzision. (Hassenstein 1979: 238)

Und:

Es ist bekannt, daß die Sprache ein Spiegel des Verstandes ist und daß die Völker, wenn sie den Verstand hoch schwingen, auch zugleich die Sprache wohl ausüben, welches der Griechen, Römer und Araber Beispiele zeigen. (Leibniz 1983: 5)

Es sind zwei Kosmopoliten, denen wir hier zuhören. 